\title{
Influência dos processos de avaliação cognitiva na atividade laboral de bombeiros portugueses
}

\author{
Rui Gomes - Universidade do Minho, Braga, Portugal \\ Fátima Teixeira - Universidade do Minho, Braga, Portugal
}

\begin{abstract}
Resumo
Este trabalho procura analisar a relação entre a atividade laboral e os processos de avaliação cognitiva em bombeiros portugueses. Participaram do estudo 163 bombeiros do distrito do Porto, em Portugal. O protocolo de avaliação incluiu medidas de avaliação cognitiva, stress, burnout, satisfação com a vida e comprometimento organizacional. Os resultados mostraram que 32,5\% dos bombeiros descreveram elevados níveis de stress ocupacional, não tendo sido assinalado nenhum caso de burnout pleno. As análises comparativas demonstraram uma experiência profissional mais negativa em profissionais que avaliaram o trabalho como mais ameaçador e menos desafiante e naqueles com menor potencial de confronto para lidar com as exigências do trabalho. As análises de regressão apontaram o valor preditivo das dimensões de stress e de avaliação cognitiva na explicação da experiência de burnout. No final do trabalho, discutem-se as implicações dos processos de avaliação cognitiva na compreensão da adaptação humana em contextos laborais.

Palavras-chave: Stress, Avaliação cognitiva, Burnout, Satisfação, Comprometimento.
\end{abstract}

Influence of cognitive appraisal processes in the work activity of firefighters

\begin{abstract}
This study analyzes the relationship between professional occupation and the processes of cognitive appraisal in Portuguese firefighters. The study involved 163 firemen from Oporto region, in Portugal. The evaluation protocol included measures of cognitive appraisal, stress, burnout, organizational commitment, and life satisfaction. The results revealed very significant levels of occupational stress (32.5\%), although no case of "full" burnout was detected. The comparative analysis showed a more negative work experience in professionals who assessed their activity as more threatening, less challenging and with a less potential for confrontation with the work demands. The regression analysis showed the predictive value of the stress dimensions and the processes of cognitive appraisal in explaining the experience of burnout. Finally, we discuss some of the implications of cognitive appraisal processes in understanding human adaptation in w orkplace settings.

Keywords: Stress, Cognitive appraisal, Burnout, Satisfaction, Commitment.
\end{abstract}

\section{Influencia de los procesos de evaluación cognitiva en la actividad laboral de los bomberos portugueses}

\begin{abstract}
Resumen
Este estudio analiza la relación entre la actividad laboral y los procesos de evaluación cognitiva en bomberos portugueses. Participaron del estudio 163 bomberos del distrito de Porto. El protocolo de evaluación incluyó medidas de evaluación cognitiva, estrés ocupacional, burnout, satisfacción con la vida y compromiso organizacional. Los resultados mostraron que el $32.5 \%$ de los bomberos presentaron altos niveles de estrés laboral aunque no se haya señalado ningún caso de burnout pleno. Los análisis comparativos revelaron experiencias laborales más negativas en aquellos profesionales que evaluaron el trabajo como más amenazante y menos desafiante y en aquellos con menor potencial para hacer frente a las exigencias del trabajo. Los análisis de regresión mostraron el valor predictivo de las dimensiones de estrés y de la evaluación cognitiva para explicar la experiencia de burnout. Se discuten las implicaciones de los procesos de evaluación cognitiva en la comprensión de la adaptación humana en contextos de trabajo.

Palabras clave: Estrés, Evaluación cognitiva, Agotamiento profesional, Satisfacción, Compromiso organizacional.
\end{abstract}

A investigação tem mostrado que os profissionais de emergência pré-hospitalar, nos quais se incluem os bombeiros, representam um dos grupos profissionais com níveis de stress mais elevados (Huynh, Xanthopoulou \& Winefield, 2013; McLennan, Birch, Cowlishaw \& Hayes, 2009). Na realidade, as tarefas inerentes à prestação de socorro (e.g., emergência médica, combate a incêndios, busca e salvamento) podem afetar a saúde e o bem-estar físico e psicológico dos bombeiros. Neste sentido, esses profissionais estão sujeitos a uma maior experiência de stress, de que são exemplos o lidar com a vida e a morte (dos próprios e dos outros), o trabalhar em situações imprevisíveis, o saber lidar com a pressão imposta pelo pouco tempo Disponivel em wnw.scielo.br para o socorro, o ter de enfrentar grandes incêndios, etc. (Marcelino \& Figueiras, 2007; Murta \& Tróccoli, 2007).

Para além da importância de compreendermos as exigências específicas que afetam esses profissionais, bem como as potenciais consequências sobre o seu funcionamento, não existem muitas indicações sobre quais os fatores que contribuem para desencadear reações mais ou menos positivas aos contextos de trabalho. A este nível, alguns autores têm vindo a realçar o papel fulcral dos processos de avaliação cognitiva na adaptação humana ao stress (Gomes, 2011; Moos \& Schaefer, 1993). É neste sentido que este trabalho se enquadra, procurando compreender a 
relação entre diferentes facetas da avaliação cognitiva e um conjunto de dimensões psicológicas associadas à atividade laboral de bombeiros portugueses (e-g., stress ocupacional, burnout, comprometimento organizacional e satisfação com a vida).

A compreensão da faceta central neste estudo (e.g., processos de avaliação cognitiva) teve por base o modelo transacional de Lazarus (1999; Lazarus \& Cohen-Charash, 2001), sendo sugerido que as reações às situações de stress devem ser vistas como um processo psicológico, onde intervêm fatores cognitivos, motivacionais e relacionais. De acordo com o autor do modelo, a experiência de stress tende a surgir quando a pessoa avalia as exigências que lhe são colocadas como superando a sua capacidade de responder e lidar com elas. Neste sentido, mais do que saber qual a frequência ou a intensidade de um episódio de stress, importa compreender o modo como cada pessoa avalia essa situação e procura resolvê-la (Lazarus \& CohenCharash, 2001).

Tendo ainda em consideração a perspetiva transacional de Lazarus (1999), a compreensão do processo de adaptação ao stress (e às emoções resultantes) relaciona-se com os fatores e etapas que lhes estão associadas. De um modo geral, perante uma situação de stress, o indivíduo começará por efetuar uma análise da relevância dessa situação para o seu bem-estar pessoal (avaliação cognitiva primária). Como refere Lazarus (2000), apenas as situações que coloquem algo em causa para o indivíduo serão passíveis de gerar uma resposta de stress a esse acontecimento. $\mathrm{O}$ resultado dessa avaliação pode significar uma percepção de ameaça (caso a situação seja percebida como demasiado exigente) ou de desafio (caso a situação seja percebida como passível de ser gerida pelo nível de capacidade da pessoa em causa). Após essa fase inicial, surge a avaliação cognitiva secundária, que basicamente diz respeito a uma análise das opções e recursos que a pessoa julga possuir para enfrentar o problema em causa. Um dos aspetos que pode condicionar a escolha da estratégia de confronto para lidar com a situação de stress, prende-se ao potencial de confronto, onde o indivíduo analisa quais os recursos que julga possuir para lidar com o problema (Lazarus, 1999).

Apesar do valor inegável dessa proposta, ainda não existe evidência suficiente acerca do impacto de cada um desses processos na adaptação ao stress. Ou seja, será que diferentes processos de avaliação cognitiva primária e secundária potenciam experiências de stress ocupacional distintas? Existirá alguma associação entre os processos de avaliação cognitiva primária e secundária? Qual ou quais dos fatores de stress e de avaliação cognitiva melhor predizem as reações dos indivíduos no trabalho?

Foi tentando responder a essas questões que este trabalho foi elaborado, procurando observar a relação entre esses processos de avaliação cognitiva e a experiência psicológica de bombeiros. Assim, para além de uma medida relacionada com a avaliação cognitiva primária e secundária, utilizaram-se também medidas destinadas a avaliar as fontes de stress sentidas pelos bombeiros na sua atividade profissional, o burnout (esgotamento), o comprometimento organizacional e a satisfação com a vida. Vários fatores sustentaram a escolha destas facetas incluídas neste trabalho.

Relativamente à medida de stress, como já foi anteriormente dito, a atividade profissional de bombeiro é bastante exigente, sendo caracterizada por diferentes fontes de pressão que podem levar a problemas de saúde física e mental (Huynh, Xanthopoulou \& Winefield, 2013; McLennan, Birch, Cowlishaw \& Hayes, 2009).

No que se refere ao burnout (esgotamento), esta dimensão foi incluída pois os bombeiros enfrentam diariamente situações emocionalmente intensas, devendo, muitas vezes, tomar decisões sob pressão, com urgência de resposta e em condições de risco ou limitação de recursos (Greenglass, Burke \& Fiksenbaum, 2001; Meyer e cols., 2012). De um modo geral, o burnout tem sido entendido como uma síndrome constituída pela exaustão emocional, despersonalização e redução da realização pessoal (Maslach, Schaufeli \& Leiter, 2001).

As outras duas áreas incluídas neste estudo representam domínios mais positivos do funcionamento humano, seja em contexto de trabalho (comprometimento organizacional), seja em termos do bem-estar individual (satisfação com a vida). Esta opção visa responder ao apelo de alguns autores no sentido de se estudar não apenas os possíveis efeitos indesejáveis do trabalho (ex: ansiedade, diminuição da autoestima, burnout, entre outros) mas também os possíveis efeitos benéficos desta atividade (ex: humor positivo, realização profissional e pessoal, entre outros) (Maslach e cols., 2001; Paton, Violanti \& Smith, 2003). Assim, no que se refere ao comprometimento organizacional, este tem sido entendido como os sentimentos, atitudes e valores positivos em relação à instituição empregadora (Mowday, Porter \& Steers, 1982), enquanto a satisfação com a vida pode ser definida como o resultado da avaliação cognitiva da pessoa acerca da sua vida, como um todo, manifestando-se pelos sentimentos de satisfação e bem-estar (Hart, 1999). Tendo por base todas essas indicações, o presente estudo procurou responder aos 
seguintes objetivos: a) identificar a experiência profissional, em termos dos níveis globais de stress, dos fatores de stress e dos índices de burnout, b) identificar as diferenças nas dimensões de stress, burnout, comprometimento organizacional e satisfação com a vida em função dos processos de avaliação cognitiva; c) identificar as variáveis preditoras da experiência de burnout.

\section{Participntes}

\section{Método}

Foi selecionada uma amostra de conveniência para este estudo com 163 bombeiros, sendo 71,7\% do sexo masculino e $28,3 \%$ do sexo feminino. As idades variaram entre os 16 e os 63 anos $(M=27,73$; $D P=10,69)$. É de assinalar que a idade de início nessa atividade pode ser relativamente precoce, estando reservadas a esses jovens atividades de aprendizagem (e menos perigosas) relacionadas com a atividade de bombeiro. No que se refere ao vínculo profissional, a grande maioria dos participantes era voluntária $(78 \%)$ e $22 \%$ eram assalariados. Na distribuição por categoria profissional, $70,7 \%$ eram socorristas, $15 \%$ eram motoristas, $10,5 \%$ eram operadores de central e 3,8\% desempenhavam a função de motorista e socorrista. $\mathrm{O}$ tempo médio de experiência profissional foi de 9,6 anos $(D P=8,85)$ e o tempo médio de trabalho por semana foi de 35.7 horas $(D P=24,75)$.

\section{Instrumentos}

Foi administrado a todos os bombeiros que participaram neste estudo um protocolo de avaliação com os instrumentos descritos abaixo. Convém realçar que as estruturas fatoriais dos instrumentos foram testadas neste estudo, utilizando-se a análise fatorial de componentes principais, sem predefinição do número de fatores, através da rotação ortogonal (procedimento varimax), com normalização de Kaiser (eigenvalue igual ou superior a um) (Tabachnick \& Fidell, 2001). Além disso, foram analisados os valores de fidelidade das dimensões de cada instrumento, através do cálculo do alpha de Crombach.

Questionário Demográfico. Para além de avaliar variáveis demográficas (ex: sexo, idade, estado civil, entre outras), procurou recolher dados relativos à formação escolar e profissional dos bombeiros (ex: habilitações literárias, vínculo profissional, horas de trabalho semanais, entre outras).

Escala de Avaliação Cognitiva (EAC). Este instrumento foi desenvolvido por Gomes (2008), tendo por base o modelo transacional de Lazarus (1999), representando uma medida da avaliação cognitiva primária e secundária dos indivíduos face à sua atividade profissional. $\mathrm{Na}$ avaliação cognitiva primária, o instrumento avalia a importância e o significado pessoal da situação em causa, sendo propostas três dimensões: i) importância (3 itens): indica até que ponto a pessoa avalia a sua ocupação como significativa do ponto de vista pessoal; ii) ameaça (3 itens): indica até que ponto a pessoa avalia a sua ocupação como perturbadora e negativa do ponto de vista pessoal; e iii) desafio (3 itens): indica até que ponto a pessoa avalia a sua ocupação como estimulante e entusiasmante do ponto de vista pessoal. $\mathrm{Na}$ avaliação cognitiva secundária, o instrumento avalia os recursos que a pessoa julga possuir para lidar com essa mesma situação e resolvê-la, sendo proposta uma dimensão: iv) potencial de confronto (3 itens): indica até que ponto a pessoa sente que possui recursos pessoais para lidar com as exigências da atividade profissional. Todos os itens são respondidos numa escala tipo likert de sete pontos (ex: $0=$ "Nada importante"; 3="Mais ou menos"; 6="Muito importante"), significando as pontuações mais altas valores mais elevados em cada uma das dimensões avaliadas. A análise realizada permitiu confirmar os quatro fatores previstos, que explicaram $75,8 \%$ da variância total. Essa estrutura fatorial teve por base um valor de KMO ("Kaiser-Meyer-Olkin") de 0,898 e um resultado significativo no teste de esferacidade de Barlett (1980.610, df 253, $\mathrm{p}=0,000)$. Os valores de fidelidade foram adequados: importância $(a=0,90)$, ameaça $(a=0,75)$, desafio $(a=0,83)$ e potencial de confronto $(a=0,87)$.

Questionário de Stress nos Prestadores de Serviços Humanos - Bombeiros (QSPSH-B). Este instrumento baseou-se no "Questionário de Stress nos Profissionais de Saúde" (QSPS), desenvolvido por Gomes, Cruz e Cabanelas (2009) com profissionais das áreas da saúde, efetuando-se as alterações necessárias de modo a adequar os itens do QSPS aos participantes neste estudo. Assim, o QSPSH-B é composto por duas partes distintas. A primeira questão avalia o nível global de stress que os profissionais experienciam no exercício da sua atividade, numa escala que varia entre zero e quatro ( $0=$ "Nenhum stress"; 4="Elevado stress"). Na segunda parte, são apresentados 25 itens relativos às fontes potencialmente geradoras de stress no desenvolvimento da sua atividade profissional, sendo avaliadas numa escala tipo likert de cinco pontos $(0=$ "Nenhum stress"; 4="Muito stress"). Foram avaliadas sete dimensões: i) lidar com clientes - referese aos sentimentos negativos dos profissionais 
relacionados com as pessoas a quem prestam os seus serviços; ii) relações profissionais - descreve o mal-estar dos profissionais relativamente ao ambiente de trabalho bem como à relação mantida com os colegas de trabalho e superiores hierárquicos; iii) excesso de trabalho - diz respeito à excessiva carga de trabalho e de horas a realizar; iv) carreira e remuneração - indica os sentimentos de mal-estar relacionados com a falta de perspetivas de desenvolvimento da carreira profissional e insatisfação com o salário recebido; v) risco de vida reporta as experiências negativas dos profissionais em situações onde podem pôr em risco a sua integridade física; vi) problemas familiares - descreve os problemas de relacionamento familiar e a falta de apoio por parte de pessoas significativas e, por fim, vii) condições de trabalho - descreve as dificuldades sentidas relacionadas com a escassez de meios humanos e materiais para a realização adequada das tarefas profissionais. Resultados mais elevados significam uma maior avaliação de stress em cada um dos domínios avaliados. A análise realizada permitiu confirmar os sete fatores previstos, mas dois itens foram eliminados devido à baixa saturação no fator em causa (6 e 19). A estrutura final explicou 76,8\% da variância total, tendo por base um valor de KMO ("Kaiser-Meyer-Olkin") de 0,898 e um resultado significativo no teste de esferacidade de Barlett (1980.610, $d f 253, \mathrm{p}=0,000)$. Os valores de fidelidade foram adequados: lidar com clientes $(a=0,86)$, relações profissionais $(a=0,85)$, excesso de trabalho $(a=0,89)$, carreira e remuneração $(a=0,73)$, risco de vida $(a=0,88)$, problemas familiares $(a=0,88)$ e condições de trabalho $(a=0,86)$.

Inventário de Burnout de Maslach-Prestadores de Serviços Humanos (IBM-PSH) (Maslach \& Jackson, 1996; Adaptação de Melo, Gomes \& Cruz, 1999). Este instrumento destina-se a avaliar os níveis de burnout (esgotamento) evidenciados por trabalhadores incluídos nas tradicionais profissões de ajuda, distribuindo-se por três dimensões: i) exaustão emocional - analisa os sentimentos de sobrecarga e exaustão emocional devido às exigências do trabalho; ii) despersonalização - mede as respostas "frias", impessoais ou mesmo negativas dirigidas àqueles a quem se prestam serviços e iii) realização pessoal avalia os sentimentos de competência profissional e de sucesso no trabalho com pessoas. O inventário é constituído por 22 itens, distribuídos pelas três dimensões referidas, sendo os itens respondidos numa escala tipo likert de 7 pontos ( $0=$ "Nunca"; 6="Todos os dias"). Os valores mais elevados significam maiores níveis de exaustão emocional, despersonalização e realização pessoal. Elevados níveis de burnout estão associados a elevados scores de exaustão emocional e despersonalização, mas também a baixos scores de realização pessoal. A análise fatorial realizada permitiu confirmar os três fatores previstos, mas três itens foram eliminados por baixa saturação no fator em causa $(9,11,21)$. A estrutura final explicou $61,6 \%$ da variância total, tendo por base um valor de $\mathrm{KMO}$ ("Kaiser-Meyer-Olkin") de 0,873 e um resultado significativo no teste de esferacidade de Barlett (1700.224, df 190, $\mathrm{p}=0,000)$. Os valores de fidelidade foram adequados: exaustão emocional $(a=0,90)$, despersonalização $(a=0,85)$ e realização pessoal $(a=0,88)$.

Escala de Comprometimento Organizacional (ECO) (Mowday e cols., 1982; Adaptação de Afonso \& Gomes, 2009). Esta escala visa avaliar os sentimentos, atitudes e valores positivos assumidos pelos profissionais relativamente ao seu local de trabalho. Os itens são respondidos numa escala tipo likert de cinco pontos (1="Discordo totalmente"; $5=$ "Concordo totalmente"), sendo possível obter um score total resultante da soma das pontuações obtidas e respetiva divisão pelo número de itens. A análise fatorial confirmou a estrutura prevista de um fator que explicou $61 \%$ da variância total, tendo por base um valor de KMO ("Kaiser-Meyer-Olkin") de 0,919 e um resultado significativo no teste de esferacidade de Barlett (888.332, df 36, $\mathrm{p}=0,000)$. O valor de fidelidade foi adequado $(a=0,92)$.

Escala de Satisfação com a Vida (ESV) (Diener, Emmons, Larsen \& Griffin, 1985; Adaptação de Neto, 1993, 1999). A escala tem por objetivo avaliar a satisfação com a vida enquanto processo cognitivo, realizando-se um julgamento pessoal acerca da vida (ex: "as minhas condições de vida são excelentes"). O instrumento é constituído por cinco itens, solicitandose às pessoas que avaliem a sua vida em geral, numa escala tipo likert de sete pontos $(1=$ "Totalmente em desacordo"; $7=$ "Totalmente em acordo"). Valores mais elevados significam maior satisfação com a vida. A análise fatorial confirmou a estrutura prevista de um fator que explicou $57,3 \%$ da variância total, tendo por base um valor de KMO ("Kaiser-Meyer-Olkin") de 0,789 e um resultado significativo no teste de esferacidade de Barlett (290.126, df 10, $\mathrm{p}=0,000)$. O valor de fidelidade foi adequado $(a=0,79)$.

\section{Procedimento}

Em todas as fases deste projeto adotaram-se atitudes e procedimentos eticamente definidos na investigação com seres humanos. Assim, foi efetuado 
um pedido formal por escrito ao Comandante de cada Corporação de Bombeiros Voluntários (todos eles na zona norte de Portugal) para a aplicação de questionários aos bombeiros das diferentes corporações, sendo apresentados os objetivos e implicações da investigação. De seguida, aplicou-se o protocolo de avaliação (que tinha um tempo médio de preenchimento de 15 minutos), tendo-se garantido o anonimato e a confidencialidade do tratamento dos dados aos participantes. Todos aqueles que desejaram fazer parte do estudo assinaram um termo de consentimento informado. No total, foram entregues 230 questionários (que corresponderam a mais de $90 \%$ dos profissionais de cada Corporação de Bombeiros), tendo sido recebidos e considerados válidos 163 questionários, representando uma taxa efetiva de retorno de $81 \% \quad(19 \%$ dos participantes não devolveram o protocolo de avaliação, decidindo não participar no estudo).

\section{Análise dos dados}

O tratamento e análise estatística dos dados foram realizados no programa SPSS 18.0 for Windows, seguindo-se diferentes procedimentos de análise. Em primeiro lugar, analisaram-se os valores médios de stress e de burnout, utilizando-se estatística descritiva das variáveis em causa. Em segundo lugar, observaram-se as diferenças na experiência de stress, burnout, comprometimento organizacional e satisfação com a vida em função da avaliação cognitiva primária e secundária, aplicando-se $t$-testes para amostras independentes nas medidas unidimensionais e análises multivariadas nas medidas multidimensionais. Paralelamente, observou-se a existência de associações entre as medidas de avaliação cognitiva primária (e.g., ameaça e desafio) e secundária (e.g., potencial de confronto), utilizando-se o teste do Qui-Quadrado, dada a transformação das dimensões da EAC em variáveis categoriais (e.g., altos e baixos valores em cada fator). Por último, utilizaram-se análises de regressão hierárquica (método "enter") para predizer a experiência de burnout (nas suas três dimensões), definindo-se como variáveis preditoras as dimensões de stress e de avaliação cognitiva. A análise dos modelos baseou-se igualmente na observação de indicadores de multicolinearidade (Índices de Tolerância, Variance Inflaction Factor e Condition Index) e de ausência de autocorrelações (Durbin-Watson), não se tendo, em geral, constatado problemas a este nível.

\section{Resultados}

\section{Estatísticas descritivas das variáveis em estudo}

Começando pelo "nível global de stress" na atividade profissional (primeira parte do QSPSH-B), é de salientar que $32,5 \%$ dos bombeiros descreveram o trabalho como sendo muito stressante (junção dos valores "bastante" e "elevado" stress da escala de likert). Relativamente aos possíveis problemas que podem contribuir para esta situação, verificou-se como principais fatores de stress as questões relacionadas com o lidar com clientes $(M=2,45, D P=0,98)$, o risco de vida $(M=2,39, D P=1,03)$, o excesso de trabalho $(M=2,31, \quad D P=2,31) \quad$ e os problemas familiares $(M=2,29, D P=1,05)$.

Para o cálculo dos níveis de esgotamento dos bombeiros, foram usadas as indicações propostas por Shirom (1989) e as adaptações subsequentes sugeridas por Gomes, Montenegro, Peixoto, e Peixoto (2010) onde se estabelecem os "pontos de corte" "uma vez por semana" da escala likert para as dimensões de exaustão emocional e despersonalização e "uma vez por mês" para a dimensão de realização pessoal. Assim, verificou-se que $8 \%$ dos bombeiros evidenciaram problemas ao nível da exaustão emocional, 8,6\% em termos de despersonalização e $9,9 \%$ demonstraram baixos índices de realização pessoal, não tendo sido detetado qualquer caso de burnout pleno.

\section{Diferenças nas dimensões psicológicas em função dos processos de avaliação cognitiva}

Nesta etapa do estudo, procurou-se verificar a importância dos processos de avaliação cognitiva primária e secundária na experiência psicológica dos bombeiros. Ou seja, questionou-se se existiriam diferenças na experiência de stress, burnout, comprometimento organizacional e satisfação com a vida em função da avaliação primária (e.g., maior ou menor tendência para avaliar o trabalho como ameaçador ou desafiante) e secundária (maior ou menor potencial de confronto). A definição do procedimento de análise foi efetuada em função do processo de confronto com o stress. Assim, a dimensão da EAC importância serviu para incluir na base de dados apenas os participantes que avaliaram o seu trabalho como minimamente significativo, tendo-se estabelecido como "ponto de corte" o valor igual ou inferior a dois para retirar os participantes das análises seguintes. Nesse caso, não foi necessário eliminar nenhum elemento da amostra. De seguida, efetuou-se a constituição dos grupos com baixos e elevados valores em cada dimensão avaliada pela EAC, definindo-se como critério de amostragem $20 \%$ de casos selecionados para cada grupo, garantindo-se que nenhum deles teria menos que 20 casos, valor este que foi significativamente superado em todas as análises 
realizadas. A Tabela 1 apresenta os resultados de todas as dimensões em que foram verificados valores significativos.

Assim, e começando pela percepção de ameaça, verificaram-se diferenças no QSPSH-B (Wilks' $\lambda=0,69$, $\left.F(7,97)=6,25, p<0,001, \eta^{2}=0,31\right)$. Os testes univariados demonstraram que os bombeiros com maior percepção de ameaça apresentaram uma experiência de stress mais elevada nas dimensões de lidar com clientes, nas relações profissionais, no risco de vida, nos problemas familiares e nas condições de trabalho. Um padrão distinto foi, no entanto, observado na experiência de burnout (Wilks' $\lambda=0,78, F(3,103)=9,94, \quad p<0,001$, $\left.\eta^{2}=0,23\right)$. Assim, foram os bombeiros com maior percepção de ameaça que evidenciaram menor despersonalização. Em termos do comprometimento organizacional e da satisfação com a vida não foram observadas diferenças significativas entre os grupos.

No que se refere à percepção de desafio, não se constataram diferenças em termos das dimensões de stress. Apesar de também não termos observado diferenças no teste multivariado do IBM-PSH (Wilks' $\left.\lambda=0,91, F(7,89)=1,29, \quad p=0,26, \eta^{2}=0,09\right)$, os testes univariados indicaram diferenças entre os grupos. Assim, os profissionais com maior percepção de desafio relataram menor exaustão emocional e despersonalização e, inversamente, maior realização pessoal. Neste mesmo sentido, estes profissionais demonstraram maior comprometimento organizacional $(t=-5,24 ; 95 ; p<0,001)$ e maior satisfação com a vida $(t=-2,94 ; 96 ; p<0,01)$.

Quanto ao potencial de confronto, o teste multivariado foi significativo no QSPSH-B (Wilks' $\left.\lambda=0,77, F(7,88)=3,74, p<0,01, \eta^{2}=0,23\right)$, tendo os testes univariados sugerido que os bombeiros com maior potencial de confronto indicaram menor stress relacionado com o lidar com clientes e com as condições de trabalho. De igual modo, observaram-se diferenças no burnout (Wilks' $\lambda=0,87, F(3,92)=4,51$, $\left.p<0,01, \eta^{2}=0,13\right)$, tendo os testes univariados apontado que os bombeiros com maior potencial de confronto percepcionaram menor exaustão emocional e despersonalização. Por último, esse mesmo grupo de profissionais assumiu maior comprometimento organizacional $(t=-4,22 ; 94 ; p<0,001)$ e maior satisfação com a vida $(t=-2,77 ; 94 ; p<0,01)$.

De seguida, analisou-se a existência de associações entre as medidas de avaliação cognitiva primária (e.g., ameaça e desafio) e secundária (e.g., potencial de confronto). Ou seja, tratava-se de saber se profissionais com tendência para percepcionarem a sua atividade como mais ou menos ameaçadora ou desafiante tendiam a perceber maior ou menor potencial de confronto para lidar com as exigências do trabalho. Assim, não foram observados valores de associação significativos entre a percepção de ameaça e o potencial de confronto. No entanto, entre a percepção de desafio e o potencial de confronto já foram observadas diferenças significativas $\left(\chi^{2}(1)=13,60, \quad p<0,001\right)$, constatando-se que o grupo com baixo desafio tendia maioritariamente a perceber um baixo potencial de confronto $(76,5 \%)$, enquanto o grupo com elevado desafio tendia maioritariamente a perceber um elevado potencial de confronto $(68,8 \%)$.

Tabela 1. Diferenças na experiência profissional em função dos processos de avaliação cognitiva

(continua)

\begin{tabular}{lccccc}
\hline \multicolumn{1}{c}{ Variável } & \multicolumn{2}{c}{ Alta ameaça } & \multicolumn{2}{c}{ Baixa ameaça } & $F$ \\
\hline QSPSH-B: Fatores de stress & $M(D P)$ & $(n)$ & $M(D P)$ & $(n)$ & $(1,103)$ \\
Lidar com clientes & & & & & \\
Relações profissionais & $2,88(0.85)$ & $(61)$ & $2,07(0.82)$ & $(44)$ & $23,86^{* * *}$ \\
Risco de vida & $2,48(0.85)$ & $(61)$ & $2,01(0.93)$ & $(44)$ & $7,31^{* *}$ \\
Problemas familiares & $2,87(0.93)$ & $(61)$ & $2,08(1.02)$ & $(44)$ & $17,11^{* * *}$ \\
Condições de trabalho & $2,56(0.94)$ & $(61)$ & $2,09(0.93)$ & $(44)$ & $6,33^{*}$ \\
IBM-PSH: Dimensões de burnout & $2,50(0.98)$ & $(61)$ & $1,97(1.06)$ & $(44)$ & $6,86^{*}$ \\
Despersonalização & & & & & $(1,105)$ \\
IBM-PSH: Dimensões de burnout & $1,14(1.39)$ & $(60)$ & $2,28(1.50)$ & $(47)$ & $16,64^{* * *}$ \\
Exaustão emocional & & & & & $(1,95)$ \\
& & & & & \\
\end{tabular}


Tabela 1. Diferenças na experiência profissional em função dos processos de avaliação cognitiva (continuação)

\begin{tabular}{|c|c|c|c|c|c|}
\hline \multirow{2}{*}{ Variável } & \multicolumn{2}{|c|}{ Alto desafio } & \multicolumn{2}{|c|}{ Baixo desafio } & \multirow[b]{2}{*}{$F$} \\
\hline & $M(D P)$ & (n) & $M(D P)$ & $(n)$ & \\
\hline Despersonalização & $0,97(0.96)$ & $(48)$ & $2,64(1,61)$ & $(49)$ & $38,06^{* * *}$ \\
\hline Realização pessoal & $4,12(1.42)$ & $(48)$ & $3,44(1,29)$ & (49) & $6,06^{*}$ \\
\hline ECO: Comprometimento organizacional & $4,28(0.66)$ & (49) & $3,45(0.89)$ & (48) & -- \\
\hline \multirow[t]{3}{*}{ ESV: Satisfação com a vida } & $4,85(1.04)$ & (49) & $4,19(1,19)$ & (49) & -- \\
\hline & \multicolumn{2}{|c|}{$\begin{array}{l}\text { Alto potencial de } \\
\text { confronto }\end{array}$} & \multicolumn{2}{|c|}{$\begin{array}{l}\text { Baixo potencial de } \\
\text { confronto }\end{array}$} & \\
\hline & $M(D P)$ & $(n)$ & $M(D P)$ & $(n)$ & $F$ \\
\hline QSPSH-B: Fatores de stress & & & & & $(1,94)$ \\
\hline Lidar com clientes & $2,02(1,07)$ & $(42)$ & $2,66(0,84)$ & (54) & $10,86^{* *}$ \\
\hline Condições de trabalho & $1,85(1,08)$ & $(42)$ & $2,40(1,36)$ & $(54)$ & $5,76^{*}$ \\
\hline IBM-PSH: Dimensões de burnout & & & & & $(1,94)$ \\
\hline Exaustão emocional & $1,72(1,22)$ & (42) & $2,56(1,19)$ & (54) & $11,63^{* *}$ \\
\hline Despersonalização & $1,22(2,26)$ & (42) & $2,02(1,61)$ & (54) & $6,94 *$ \\
\hline ECO:Comprometimento organizacional & $4,27(0,67)$ & (43) & $3,57(0,90)$ & (53) & -- \\
\hline ESV: Satisfacão com a vida & $4,87(1,26)$ & (43) & $4,19(1,14)$ & (53) & -- \\
\hline
\end{tabular}

${ }^{*} p<0,05 ;{ }^{* *} p<0,01 ; * * * p<0,001$

\section{Predição da experiência de burnout}

Nesta última parte do trabalho, analisou-se até que ponto as dimensões da avaliação cognitiva (EAC) e do questionário de stress (QSPSH-B) poderiam contribuir para a explicação das três dimensões de Burnout avaliadas pelo IBM-PSH. Assim, nos modelos de regressão as variáveis foram introduzidas pela seguinte ordem: i) fatores de stress do QSPSH-B, ii) dimensão de importância da EAC, iii) dimensões de ameaça e de desafio da EAC, e iv) dimensão de potencial de confronto da EAC. Esta entrada das variáveis nos modelos de regressão procurou refletir a natureza do processo de confronto com o stress, preconizando-se o início do "encontro transacional" com as fontes específicas de pressão, seguindo-se a significação pessoal atribuída a essa situação (importância), o resultado da avaliação cognitiva primária (ameaça e desafio) e, por fim, a avaliação cognitiva secundária (potencial de confronto).

Assim sendo, e começando pela predição da exaustão emocional, o primeiro bloco de entrada permitiu explicar $32 \%$ da variância. Nesse caso, a exaustão emocional foi predita por maiores níveis de stress associados à carreira e remuneração e, com valores quase significativos, pelas relações profissionais e pelo excesso de trabalho. $O$ segundo bloco de entrada aumentou a percentagem de variância para $38 \%$, verificando-se que a exaustão emocional foi predita por uma menor percepção de importância. A introdução da ameaça e desafio colocou o modelo com $42 \%$ de variância explicada, sendo a exaustão emocional predita pela menor percepção de desafio. No quarto, e último bloco, o modelo passou a explicar $44 \%$ da variância, sendo a exaustão emocional predita por menores níveis de potencial de confronto.

Ao nível da despersonalização, o primeiro bloco de entrada explicou $10 \%$ de variância, sendo a despersonalização explicada pelo menor stress associado ao lidar com clientes e, inversamente, pelo maior stress relacionado com a carreira e remuneração. No segundo bloco de entrada, o modelo passou a predizer $24 \%$ de variância, sendo a despersonalização predita por uma menor percepção de importância. No terceiro bloco, o modelo passou a predizer $40 \%$ de variância, sendo a despersonalização predita por uma menor percepção de ameaça e de desafio. No último bloco, não se observaram valores significativos no potencial de confronto.

Finalmente, a maior realização pessoal foi explicada, no primeiro bloco de entrada, pelo maior stress associado ao lidar com clientes, explicando $13 \%$ de variância. Esse valor aumentou para o segundo bloco de entrada, com $24 \%$ de variância explicada, sendo a maior realização pessoal explicada por uma maior percepção de importância. Os terceiro e quarto blocos de entrada não se revelaram significativos.

Os valores obtidos nos três modelos de regressão podem ser consultados na Tabela 2 . 
Tabela 2. Modelos de regressão para a predição do burnout

\begin{tabular}{|c|c|c|c|c|}
\hline Exaustão emocional & $R^{2}$ (R $R^{2}$ ajust. $)$ & F & $\beta$ & $t$ \\
\hline Bloco 1: QSPSH-B: Fatores de stress & & $(7,149)$ & & \\
\hline Relações profissionais & & $11,61 * * *$ & 0,21 & $1,90^{+}$ \\
\hline Excesso de trabalho & & & 0,20 & $1,91^{+}$ \\
\hline Carreira e remuneração & $0,35(0,32)$ & & 0,23 & $2,68^{* *}$ \\
\hline Bloco 2: EAC: avaliação primária & & $(8,148)$ & & \\
\hline Importância & $0,41(0,38)$ & $13,08^{* * *}$ & $-0,25$ & $-3,94 * * *$ \\
\hline Bloco 3: EAC: avaliação primária & & $(10,146)$ & & \\
\hline Ameaça & & $12,47 * * *$ & $-0,01$ & n.s. \\
\hline Desafio & $0,46(0,42)$ & & $-0,26$ & $-3,32 * *$ \\
\hline Bloco 4: EAC: avaliação secundária & & $(11,145)$ & & \\
\hline Potencial de confronto & $0,48(0,44)$ & $11,98^{* * *}$ & $-0,17$ & $-2,07 *$ \\
\hline Despersonalização & $R^{2}$ ( $R^{2}$ ajust.) & $F$ & $\beta$ & $t$ \\
\hline Bloco 1: QSPSH-B: fatores de stress & & $(7,147)$ & & \\
\hline Lidar com clientes & & $3,43^{* *}$ & $-0,31$ & $-2,61 *$ \\
\hline Carreira e remuneração & $0,14(0,10)$ & & 0,25 & $2,50^{*}$ \\
\hline Bloco 2: EAC: avaliação primária & & $(8,146)$ & & \\
\hline Importância & $0,28(0,24)$ & $7,07 * * *$ & $-0,39$ & $-5,36 * * *$ \\
\hline Bloco 3: EAC: avaliação primária & & $(10,144)$ & & \\
\hline Ameaça & & $11,14^{* * *}$ & $-0,24$ & $-3,23 * *$ \\
\hline Desafio & $0,44(0,40)$ & & $-0,37$ & $-4,44 * * *$ \\
\hline Bloco 4: EAC: avaliação secundária & & $(11,143)$ & & \\
\hline Potencial de confronto & $0,44(0,40)$ & $10,29 * * *$ & 0,10 & n.s. \\
\hline Realização pessoal & $R^{2}$ ( $R^{2}$ ajust.) & $F$ & $\beta$ & $t$ \\
\hline Bloco 1: QSPSH-B: fatores de stress & & $(7,148)$ & & \\
\hline Lidar com clientes & $0,17(.13)$ & $4,26^{* * *}$ & 0,34 & $2,85^{* *}$ \\
\hline Bloco 2: EAC: avaliação primária & & $(8,147)$ & & \\
\hline Importância & $0,28(.24)$ & $6,98^{* * *}$ & 0,34 & $4,67 * * *$ \\
\hline Bloco 3: EAC: avaliação primária & & $(10,145)$ & & \\
\hline Ameaça & & $5,55^{* * *}$ & $-0,02$ & n.s. \\
\hline Desafio & $0,28(.23)$ & & $-0,04$ & n.s. \\
\hline Bloco 4: EAC: avaliação secundária & & $(11,144)$ & & \\
\hline Potencial de confronto & $0,28(.22)$ & $5,01 * * *$ & $-0,02$ & n.s. \\
\hline
\end{tabular}

n.s. =não significativo; $\quad{ }^{+} p<0,10 ;{ }^{*} p<0,05 ;{ }^{*} p<<0,01 ;{ }^{* * *} p<0,001$

\section{Discussão}

A atividade de profissionais ligados a missões de risco, como é o caso dos bombeiros, pode levá-los a experienciar níveis de stress ocupacional que afetam a sua qualidade de vida, bem como dar origem a reações pós-traumáticas e potenciar os sentimentos de burnout (Benedek, Fullerton \& Ursano, 2007; Brough, 2004). A este nível, tem sido evidente uma preocupação da literatura em estudar os possíveis efeitos negativos do stress laboral, existindo contudo uma menor tendência para compreender os processos psicológicos que estão na base de experiências de trabalho potencialmente desajustadas ou satisfatórias.

É precisamente neste âmbito que este trabalho se insere, procurando não apenas compreender os níveis de stress laboral e de burnout dos bombeiros, mas sobretudo analisar a influência dos processos de avaliação cognitiva no modo como esses profissionais vivenciam o seu trabalho.

Começando pela experiência de stress, dois dados merecem ser destacados. Como seria de esperar, o 
primeiro aspeto demonstra que esta atividade é passível de gerar níveis significativos de stress numa percentagem assinalável de bombeiros (mais de 30\% dos participantes), acabando por colocá-los ao nível dos valores observados na área do ensino (Gomes $e$ cols., 2010; Pinto, Lima \& Silva, 2005), da saúde (Silva \& Gomes, 2009) e da segurança pública (Afonso \& Gomes, 2009; Maslach-Pines \& Keinan, 2006). O segundo ponto, prende-se com os fatores de stress, realçando-se essencialmente a pressão associada ao lidar com clientes e o risco de vida para o próprio profissional. Esses dados revelam a natureza central desta atividade, dadas as relações interpessoais eminentemente exigentes deste trabalho, a que acresce noutras ocasiões a exposição a situações de perigo para a integridade física e psicológica do bombeiro. Nesse sentido, pode-se afirmar que o instrumento de stress utilizado neste estudo refletiu integralmente as dimensões fundamentais que definem esta atividade. Apesar de podermos encarar esses fatores como inerentes à atividade dos bombeiros, a verdade é que a repetição cumulativa a situações de risco pode contribuir para o desenvolvimento da perturbação de stress pós-traumático (Jaycox \& Foa, 1998) devendo, por isso, merecer a devida atenção em termos do acompanhamento e apoio dado a esses profissionais.

No que concerne aos níveis de burnout, os dados também se enquadram nos encontrados noutras profissões (ver Bezerra \& Beresin, 2009; Gomes e cols., 2010; Silva \& Gomes, 2009), sendo talvez de destacar os valores mais acentuados na despersonalização (praticamente 9\% dos participantes), que é um valor semelhante ao encontrado por Afonso e Gomes (2009) em profissionais de segurança pública.

Quanto ao papel dos processos de avaliação cognitiva na experiência de stress, comprometimento organizacional e satisfação com a vida, os resultados foram uniformes no salientar da sua relevância junto desses profissionais. Tal como esperado face à proposta conceptual utilizada, os bombeiros com uma percepção de ameaça mais elevada relataram maior stress em cinco fatores do QSPSH-B (e.g., lidar com clientes, relações profissionais, risco de vida, problemas familiares e condições de trabalho) a que juntou, no entanto, uma menor tendência para a despersonalização no IBM-PSH. Já os profissionais com uma maior percepção de desafio indicaram menores níveis de exaustão emocional e despersonalização e maiores níveis de realização pessoal no IBM-PSH. De igual modo, esse grupo também apontou maior comprometimento organizacional e satisfação com a vida. Ao nível da avaliação cognitiva secundária, os bombeiros com maior potencial de confronto, apresentaram menor stress ocupacional relativamente ao lidar com clientes e às condições de trabalho, menor burnout na exaustão emocional e na despersonalização, a que se juntou também um maior comprometimento organizacional e uma maior satisfação com a vida. Dito de forma mais resumida, fica claro neste estudo o impacto dos processos de avaliação cognitiva na experiência profissional dos bombeiros, confirmando-se assim a necessidade de atendermos a esses aspetos em estudos futuros, tal como proposto no modelo transacional (ver Lazarus, 1999, 2000).

Nesse mesmo sentido, os modelos de regressão tornaram patente o valor preditivo da avaliação cognitiva na explicação da experiência de burnout. A este nível, repare-se que ao introduzirmos as facetas de avaliação cognitiva primária e, com menor impacto, o potencial de confronto, as percentagens de variância explicada subiram significativamente, o que traduz a importância de considerar estes domínios para se compreender melhor o problema do burnout. Esta situação foi particularmente evidente na predição da despersonalização, que obteve $14 \%$ de variância explicada com as variáveis de stress e finalizou com $40 \%$ de variância final explicada, sendo um valor bastante acima dos obtidos noutros estudos (Afonso \& Gomes, 2009; Gomes e cols., 2009, 2010).

Devemos ainda referir que alguns dos dados obtidos neste estudo merecem clarificação em investigações futuras, nomeadamente a possibilidade dos níveis superiores de ameaça e de stress relacionado com clientes implicaram menor tendência para a despersonalização. De alguma maneira, para esses profissionais, quanto maior a pressão nestes domínios, maior é a sua focalização na pessoa a quem prestam os seus serviços, podendo justificar-se estes resultados pelo sentimento de ajuda desta atividade. Por outro lado, será interessante testar em estudos futuros a interação entre a avaliação cognitiva primária e secundária, adicionando-se a esta última área outras facetas de avaliação (ex: perceção de controle, expetativas futuras, entre outras). Relativamente às limitações deste trabalho, devemos referir a utilização de uma metodologia de investigação transversal (que não permite estabelecer relações causais entre as variáveis em estudo) bem como a utilização de uma amostra de conveniência, que obviamente não é representativa da população em estudo. No entanto, independentemente desses aspetos, ficou evidente a relação entre as variáveis em análise e o impacto dos processos de avaliação cognitiva na experiência profissional dos bombeiros, sendo este um dos objetivos principais deste estudo.

Em síntese, os dados obtidos indicaram a importância de se estudar o stress ocupacional nos 
bombeiros, uma vez que este problema está longe de ser meramente residual, e, por outro lado, apontaram o valor explicativo dos processos de avaliação cognitiva no modo como os profissionais vivenciam a sua atividade profissional.

\section{Referências}

Afonso, J. M. P. \& Gomes, A. R. (2009). Stress ocupacional em profissionais de segurança pública: um estudo com militares da Guarda Nacional Republicana. Psicologia: Reflexão e Crítica, 22(2), 294303.

Benedek, D. M., Fullerton, C. \& Ursano, R. J. (2007). Rescue workers: mental health consequences of natural and human-made disasters for public health and public safety workers. Annual Review of Public Health, 28, 55-68.

Bezerra, R. P. \& Beresin, R. (2009). A síndrome de burnout em enfermeiros da equipe de resgate préhospitalar. Einstein, 7(3), 351-356.

Brough, P. (2004). Comparing the influence of traumatic and organizational stressors on the psychological health of police, fire, and ambulance officers. International Journal of S tress Management, 11, 227-244.

Diener, E., Emmons, R., Larsen, R. \& Griffin, S. (1985). The satisfaction with life scale. Journal of Personality Assessment, 49, 71-75.

Gomes, A. R. (2008). Escala de Avaliação Cognitiva. Relatório técnico não publicado. Braga: Instituto de Educação e Psicologia, Universidade do Minho.

Gomes, A. R. (2011). Adaptação humana em contextos desportivos: contributos da teoria para a avaliação psicológica. Avaliação Psicológica, 10(1), 13-24.

Gomes, A. R., Cruz, J. F. \& Cabanelas, S. (2009). Estresse ocupacional em profissionais de saúde: Um estudo com enfermeiros portugueses. Psicologia: Teoria e Pesquisa, 25(3), 307-318.

Gomes, A. R., Montenegro, N., Peixoto, A. B. \& Peixoto, A. R. (2010). Stress ocupacional no ensino: um estudo com professores dos $3^{\circ}$ ciclo e ensino secundário. Psicologia \& Sociedade, 22(3), 587-597.

Greenglass, E. R., Burke, R. J. \& Fiksenbaum, L. (2001). Workload and burnout in nurses. Journal of Community and Applied Social Psychology, 11, 211-215.

Hart, P. (1999). Predicting employee life satisfaction: a coherent model of personality, work and nonw ork experiences, and domain satisfactions. Journal of Applied Psychology, 84, 564-584.

Huynh, J. Y., Xanthopoulou, D. \& Winefield, A. H. (2013). Social support moderates the impact of demands on burnout and organizational connectedness: a two-wave study of volunteer firefighters. Journal of Occupational Health Psychology, 18(1), 9-15.

Jaycox, L. H. \& Foa, E. B. (1998). Post-traumatic stress disorder. Em A. S. Bellack \& M. Hersen (Eds.). Comprehensive clinical psychology (pp. 499-517). Nova Iorque: Elsevier Science.

Lazarus, R. S. (1999). Stress and emotion: a new synthesis. Nova Iorque: Springer.

Lazarus, R. S. (2000). How emotions influence performance in competitive sports. The Sport Psychologist, 14, 229-252.

Lazarus, R. S. \& Cohen-Charash, Y. (2001). Discrete emotions in organizational life. Em R. L. Payne and C. L. Cooper (Eds.). Emotions at work: theory, research and applications for management (pp. 45-81). Chichester: John Wiley \& Sons, Ltd.

Marcelino, D. \& Figueiras, M. J. (2007). A perturbação pós-stress traumático nos socorristas de emergência pré-hospitalar: influência do sentido interno de coerência e da personalidade. Psicologia, Saúde \& Doenças, 8(1), 95-108.

Maslach, C. \& Jackson, S. E. (1996). Maslach Burnout Inventory - Human Services Survey (MBI-HSS). Em C. Maslach, S. E. Jackson \& M. P. Leiter (Eds.). MBI Manual (3 ed., pp. 3-17). Mountain View, CA: CPP, Inc.

Maslach, C., Schaufeli, W. B. \& Leiter, M. P. (2001). Job burnout. Annual Review of Psychology, 52, $397-$ 422.

Maslach-Pines, A. \& Keinan, G. (2006). Stress and burnout in israeli border police. International Journal of Stress Management, 13, 519-540.

McLennan, J., Birch, A., Cowlishaw, S. \& Hayes, P. (2009). Maintaining volunteer firefighter numbers: adding to value to the retention coin. Australian Journal of Emergency Management, 24, 40-47.

Melo, B. T., Gomes, A. R. \& Cruz, J. F. (1999). Desenvolvimento e adaptação de um instrumento de avaliação psicológica do burnout para os profissionais de psicologia. Em A. P. Soares, S. Araújo \& S. Caires (Eds.). Avaliação psicológica: Formas e contextos (vol. VI, pp. 596-603). Braga: APPORT (Associação dos Psicólogos Portugueses) - Universidade do Minho.

Meyer, E. C., Zimering, R., Daly, E., Knight, J., Kamholz, B. W. \& Gulliver, S. B. (2012). Predictors of posttraumatic stress disorder and other psychological symptoms in trauma-exposed firefighters. Psychological Services, 9(1), 1-15.

Moos, R. H. \& Schaefer, J. A. (1993). Coping resources and processes: current concepts and measures. Em L. Goldberger \& S. Breznitz (Eds.). Handbook of 
stress: Theoretical and clinical aspects ( $2^{\mathrm{a}}$ ed., pp. 234257). Nova Iorque: Free Press.

Mowday, R. T., Porter, L. W. \& Steers, R. M. (1982). Employee-organization linkages. The psychology of commitment, absenteeism, and turnover. Nova Iorque: Academic Press.

Murta, S. G. \& Tróccoli, B. T. (2007). Stress ocupacional em bombeiros: efeitos de intervenção baseada em avaliação de necessidades. Estudos de Psicologia, 24(1), 41-51.

Neto, F. (1993). Satisfaction with life among Portuguese adolescents. Journal of Youth and Adolescence, 22, 125-134.

Neto, F. (1999). Satisfação com a vida e características da personalidade. Psychologica, 22, 55-70.

Paton, D., Violanti, J. M. \& Smith, L. M. (Eds.). (2003). Promoting capabilities to manage post-traumatic stress: Perspectives on resilience. Springfield, Il: Charles C Thomas.

Pinto, A. M., Lima, M. L. \& Silva, A. L. (2005). Fuentes de estrés, burnout y estrategias de coping en profesores portugueses. Revista de Psicologia del Trabajo y de las Organizaciones, 21, 125-143.

Silva, M. \& Gomes, A. R. (2009). Stress ocupacional em profissionais de saúde: um estudo com médicos e enfermeiros portugueses. Estudos de Psicologia (Natal). 14(3), 239-248.

Shirom, A. (1989). Burnout in work organizations. Em C. L. Cooper \& I. Robertson (Eds.). International review of industrial and organizational psychology (pp. 2548). Nova Iorque: Wiley.

Tabachnick, B.G. \& Fidell, L.S. (2001). Using Multivariate Statistics $\left(4^{\mathrm{a}}\right.$ ed.). Nova Iorque: HarperCollins.
Recebido em 22/10/2011

Reformulado em 05/12/2012

Aprovado em 14/05/2013

Sobre os autores:

Rui Gomes é professor auxiliar da Escola de Psicologia da Universidade do Minho, Portugal. Obteve a Licenciatura em Psicologia, Área de Psicologia Social, Comunitária e das Organizações pela Universidade do Minho. Tem Mestrado em Psicologia, Especialização em Psicologia Clínica, pela Universidade do Minho e Doutoramento em Psicologia, área de conhecimento em Psicologia do Desporto pela Universidade do Minho.

Fátima Teixeira é licenciado em Enfermagem, exercendo a sua atividade profissional como enfermeira em instituições de saúde no norte de Portugal. Em 2010, concluiu o Mestrado Integrado em Psicologia, Área de Especialização de Psicologia Clínica na Escola de Psicologia da Universidade do Minho.

Contato com os autores:

Rui Gomes

Universidade do Minho - Escola de Psicologia

Campus de Gualtar - CEP 4710-057 - Braga, Portugal.

E-mail: rgomes@psi.uminho.pt 